\title{
Clinicopathologic features and treatment of breast metastasis from nasopharyngeal carcinoma: A report of two cases and literature review
}

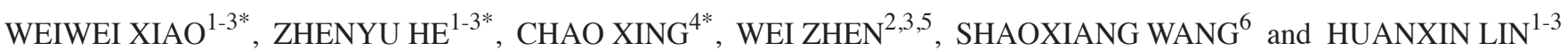 \\ ${ }^{1}$ Department of Radiotherapy, Sun Yat-Sen University Cancer Center; ${ }^{2}$ State Key Laboratory of Oncology in South China; \\ ${ }^{3}$ Collaborative Innovation Center of Cancer Medicine, Guangzhou, Guangdong 510060; ${ }^{4}$ Department of Oncology, \\ Tengzhou Center People's Hospital, Tengzhou, Shandong 277500; ${ }^{5}$ Department of Ultrasonography, \\ Sun Yat-Sen University Cancer Center, Guangzhou, Guangdong 510060; ${ }^{6}$ Institute of Molecular Medicine, \\ Medical School of Shenzhen University, Shenzhen University, Shenzhen, Guangdong 518000, P.R. China
}

Received September 3, 2014; Accepted June 16, 2015

DOI: $10.3892 / \mathrm{ol} .2015 .3818$

\begin{abstract}
Breast metastasis is a rare pattern of distant metastasis from nasopharyngeal carcinoma (NPC) following curative treatment. The present study reported two patients with breast metastasis from NPC and reviewed four such patients reported in the literature, including the analysis of clinical profiles, pathological diagnoses, treatment strategies and outcomes. The breast is a potential metastatic site for patients with NPC following curative treatment; however, thus far, only metachronous breast metastasis has been reported. Infection with Epstein-Barr virus (EBV) was identified in the breast metastasis sites of the two patients investigated in the current study using in situ hybridization for EBV-encoded small RNAs. Patients with breast metastases from NPC consistently and simultaneously develop metastases in other sites. In addition, the two current patients had poor prognoses. Therefore, chemotherapy was the primary treatment strategy administered. A solitary breast metastasis was identified in the male patient. At the most recent follow-up 10 years subsequent to undergoing a Halsted mastectomy, the patient remained alive, however the patient is now lost to follow-up. This case indicates that patients exhibiting NPC with a solitary breast metastasis may still gain long-term survival by undergoing
\end{abstract}

Correspondence to: Professor Huanxin Lin, Department of Radiotherapy, Sun Yat-Sen University Cancer Center, 651 East Dongfeng Road, Guangzhou, Guangdong 510060, P.R. China

E-mail: linhx@sysucc.org.cn

*Contributed equally

Key words: nasopharyngeal carcinoma, breast metastasis, epstein-barr virus radical mastectomy; therefore, radical mastectomy should be considered in such patients.

\section{Introduction}

Although breast cancer is the most common malignancy among female individuals, the breast is an uncommon site of metastasis from extramammary malignant neoplasms. In addition, metastases to the breast represent only $0.3-2.7 \%$ of all malignant mammary tumours (1). Excluding contralateral mammary tumours, the most common sources of primary tumour metastasis to the breast are, in decreasing order of frequency, melanoma, lymphoma, lung and ovarian cancer (2). Metastases from the head and neck cancer are rare, and those from nasopharyngeal carcinoma (NPC) are extremely rare (2).

Diagnosis of breast metastasis from NPC is difficult, even when the patient has a medical history of another primary cancer. Experience of dealing with this clinical setting is lacking; in fact, only four documented cases have been reported in the literature thus far (3-5), and comprehensive descriptions of the biological behavior and optimal treatment of this entity are lacking. To reveal important aspects of breast metastasis from NPC, the present study reports two cases from the Sun Yat-Sen University Cancer Center (SYSUCC; Guangzhou, China) and reviews previous case reports of breast metastasis from NPC, emphasizing the clinicopathological and radiological features, diagnosis, treatment strategies, and outcomes.

To the best of our knowledge, the current case study describes the only case of a male patient in this clinical setting exhibiting long-term survival after undergoing salvage treatment. In addition, this is the first study to review all available cases of breast metastasis from NPC.

\section{Case report}

Patients. From the pathology files of the SYSUCC (Guangzhou, China), two cases of breast metastasis from NPC were identified. Clinical data, including patient information, tumour characteristics, treatment and outcomes, were 
collected. Informed consent was obtained from the patients at their initial visit for the collection of clinical information. The review, analysis and publication of the current data were approved by the Research Ethics Board of SYSUCC. Tumour response to treatment was assessed according to the Response Evaluation Criteria in Solid Tumours (6), and survival was traced through medical records and phone interviews.

Pathological analysis. Immunohistochemistry (IHC) was performed on paraffin-embedded sections using the avidin-biotin-peroxidase complex method (7), with antibodies for human estrogen receptor (ER), progesterone receptor (PR) and human epidermal growth factor receptor 2 (HER2; Santa Cruz Biotechnology, Inc., Santa Cruz, CA, USA). In situ hybridization (ISH) for Epstein-Barr virus (EBV)-encoded small RNAs (EBER) was performed on paraffin-embedded sections, according to the manufacturer's instructions (Dako, Glostrup, Denmark). The samples were obtained from lumpectomy for case 1 and halsted mastectomy in case 2 . Briefly, $4-\mu$ m paraffin embedded sections were deparaffinized, rehydrated, and predigested with proteinase K. A fluorescein-conjugated EBER probe was applied and the sections were incubated at $37^{\circ} \mathrm{C}$ for $2 \mathrm{~h}$. Subsequently, alkaline phosphatase-conjugated antibody to fluorescein was applied, followed by chromogen. Sections were then counterstained with hematoxylin (Sigma-Aldrich, St. Louis, MO, USA). Dark brown staining of the cell nucleus was regarded as positive expression.

Case 1. A 41-year-old female patient presented in July 2008 to Sun Yat-Sen University Cancer Center (Shenzen, China) with a 1-month history of left upper neck swelling, tinnitus and epistaxis. Physical examination revealed a mass in the nasopharynx and bilateral enlargement of the jugular-carotid lymph nodes. The size of the lymph node at the left upper neck was 7x6 cm and the diameter of the lymph node in the right neck was $1.5 \mathrm{~cm}$. Analysis of biopsies obtained from the growth in the nasopharynx were used to determine a diagnosis of poorly differentiated squamous cell carcinoma. Magnetic resonance imaging scans of the head and neck revealed tumour infiltration of the roof and lateral walls, and the skull base. No distant metastasis was identified upon initial diagnosis and the patient was classified with stage T3N3M0 NPC (8). The patient was treated with one cycle of cisplatin $\left(80 \mathrm{mg} / \mathrm{m}^{2}\right.$ on day 1$)$ and 5-fluorouracil (5-FU; $500 \mathrm{mg} / \mathrm{m}^{2} /$ day on days $\left.1-5\right)$ regimen followed by curative two-dimensional radiotherapy. The patient received doses of $70 \mathrm{~Gy}$ in 35 fractions to the primary tumour, 66 Gy in 33 fractions to the right neck and 62 Gy in 31 fractions to the left neck. Chemotherapy was performed between 25 July 2008 to 1 August 2008. Radiotherapy was performed between 12 August 2008 and 30 September 2008. Complete remission was achieved after radiotherapy was finished. Upon termination of treatment, complete remission was achieved.

In January 2009, the patient presented with a mass in the right breast. Physical examination revealed a firm mass in the lateral upper quadrant of the right breast measuring $1.5 \mathrm{~cm}$ in diameter. No mass was identified in the left breast or bilateral axillary fossa.

Ultrasonography identified a hypoechoic lesion with a smooth margin in the lateral upper quadrant of the right breast, and no retrotumour acoustic shadowing, microcalcification, skin change or obvious blood signal, as indicated in Fig. 1A. Furthermore, no obvious lymph node enlargement was detected in the two auxiliary fossa. Concurrently, multiple diffuse hypoechoic lesions with smooth margins were identified in the liver, as demonstrated in Fig. 1B. Chemical profiling revealed anemia (88 g/l Hb; normal range, 130-160 g/1 Hb) and enzyme elevation, including alanine aminotransferase (115.1 U/1; normal range, 0.0-40.0 U/1), alkaline phosphatase (253.3 U/1; normal range, 0.0-45.0 U/1), $\gamma$-glutamyl transferase (226.4 U/1; normal range, 11.0-50.0 U/1) and lactate dehydrogenase (1,328.2 U/1; normal range, 109.0-245.0 U/1). In addition, carcinoembryonic antigen levels (CEA; $74.4 \mathrm{ng} / \mathrm{ml}$; normal range, $0-5 \mathrm{ng} / \mathrm{ml}$ ) were notably higher than the normal range. Prior to characterisation of the breast mass, a diagnosis of liver metastasis was considered. Thus, a segmental resection (lumpectomy) was performed on the breast on January 20, 2009. Histological analysis performed on the metastatic breast tissue demonstrated malignant anaplastic carcinoma proliferating in a nested and trabecular pattern (Fig. 2A), and neoplastic cells exhibited a short spindle or round appearance with little cytoplasm (Fig. 2B). Immunostaining results were as follows: ER (-), PR (-) and HER2 (-) (Fig. 2C-E). ISH determined that the neoplastic cells were positively labeled with the EBER probe (Fig. 2F).

Palliative care with chemotherapy was planned, however, the patient refused further treatment, was discharged from hospital on 25 January 2009 and succumbed to cachexia 5 months after leaving hospital.

Case 2. A 46-year-old male patient presented in October 2000 to Sun Yat-Sen University Cancer with a 6-month history of nasal and 1-month history of right neck swelling. Physical examination identified a mass in the roof of the nasopharynx and a lump in the right neck. The size of lymph node at the right side was $8 \times 4 \mathrm{~cm}$. Computed tomography (CT) scan of the nasopharynx indicated tumour infiltration of the lateral wall and roof of the nasopharyngeal cavity, bilateral walls of the oropharynx, and the two posterior portions of the nasal cavity, as well as invasion of the skull base, right maxillary sinus and sphenoid sinus. No distant metastasis was identified on chest X-ray, abdominal ultrasonography or emission CT examination. Thus, the patient was diagnosed with T4N3M0 stage IV NPC. Prior to radiotherapy, one cycle of PF regimen chemotherapy (30 mg cisplatin for 5 days plus $750 \mathrm{mg}$ floxuridine for 5 days) was administered to the patient. Curative radiotherapy was performed between November 2000 and January 2001. The dose to the primary tumour was $74 \mathrm{~Gy}$ in 37 fractions, to the right neck was 70 Gy in 35 fractions and to the left neck was 60 Gy in 30 fractions. At the termination of treatment the size of the residual lymph node at the right side had reduced to $1.5 \times 1.5 \mathrm{~cm}$ and, 3 months after treatment, complete remission was achieved.

In July 2001, the patient identified a palpable mass in the left breast and returned to Sun Yat-Sen University Cancer for a consultation. Physical examination revealed a mass measuring $3 \times 2 \mathrm{~cm}$ in the lateral upper quadrant of the left breast. Fine-needle biopsy identified a small number of atypical cells, with an appearance of bare and obvious nucleus. 


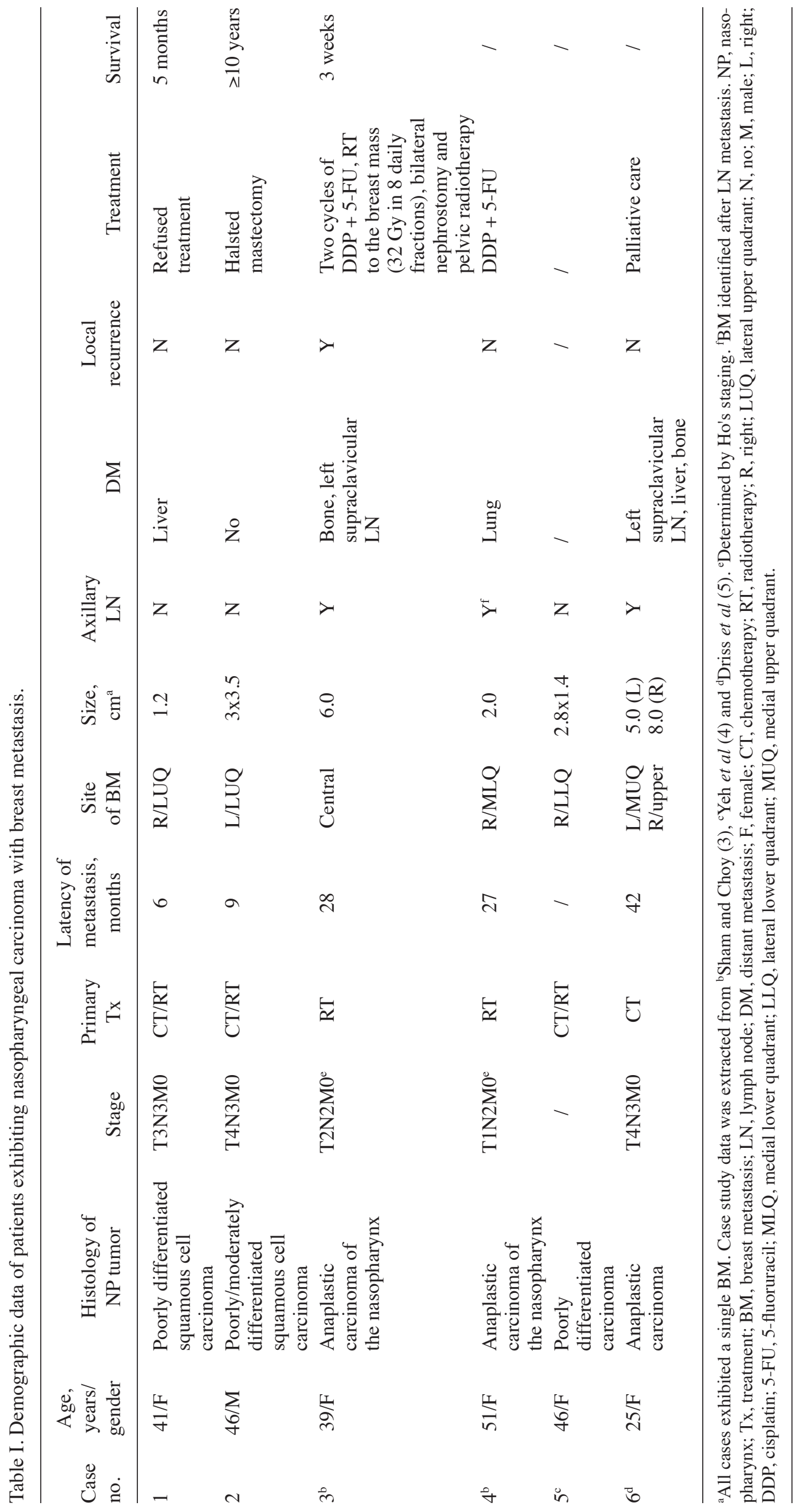


A

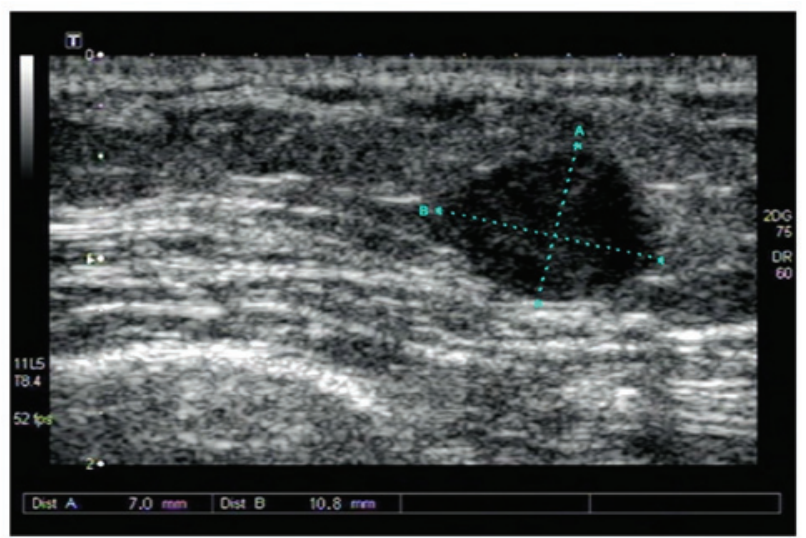

B

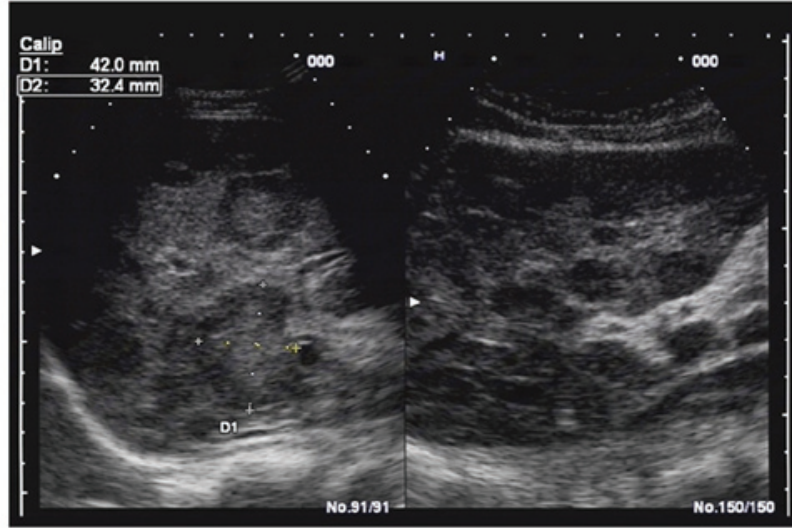

Figure 1. Ultrasonography scans of breast and liver metastases in a patient with nasopharyngeal carcinoma following curative treatment. (A) Hypoechoic lesion in the lateral upper quadrant of the right breast with a smooth margin. (B) Multiple diffuse hypoechoic lesions with smooth margins located in the liver.

Metastasis from the nasopharynx was suspected. Blood profiling identified elevation of EBV-DNase antibodies from $49 \%$ at diagnosis to $71 \%$. However, CEA $(0.1 \mathrm{ng} / \mathrm{ml}$; normal range, $0-5 \mathrm{ng} / \mathrm{ml}$ ) and squamous cell carcinoma antigen $(0.3 \mathrm{ng} / \mathrm{ml}$; normal range, $0-1.5 \mathrm{ng} / \mathrm{ml})$ levels were normal, and there was no evidence of distant metastasis in the lung or abdomen.

A solitary metastatic lesion was identified in the breast. Such patients have potentially good prognoses, therefore, a Halsted mastectomy was performed on the left breast on 10 August 2001. Histological analysis revealed poorly and moderately differentiated squamous cell carcinoma diffusely infiltrated in the left breast and pectoralis muscles (Fig. 3A and B). Immunostaining results were as follows: ER (-), PR (-) and HER2 (-) (Fig. 3C-E). ISH revealed that the neoplastic cells were positively labeled with an EBER probe (Fig. 3F). No tumour cells were observed in the axillary lymph nodes.

The patient was disease-free and without discomfort at the most recent follow-up on May 8, 2010, but was then lost to follow-up.

\section{Literature review}

Search strategy. The PubMed database was searched for all English literature publications using the following keywords: 'Breast metastasis' 'breast metastases' 'metastases to the breast' 'metastasis to the breast' 'metastasis in the breast' 'metastases in the breast' and 'nasopharyngeal carcinoma' and 'nasopharyngeal cancer'. Four cases of breast metastasis from NPC were identified and are included in the current analysis, termed cases 3-6 (2-4). The six cases, including cases 1 and 2 of the present study, are summarized in Table I and discussed hereafter.

Clinical manifestation. Five of the six patients were female while case 2 from the current series was male. The age at initial diagnosis of NPC ranged between 25 and 51 years, with a median age of 43.5 years. The primary staging classifications of five of the documented patients were all loco-regionally advanced without distant metastasis. Radiotherapy with or without chemotherapy was administered in cases 1-5; however, case 6 received chemotherapy alone. Following a latency period of 6-42 months, patients were diagnosed with metastasis in the breast (median latency period, 27 months). The predominant symptoms at relapse were the presence of a breast mass and axillary lymph node enlargement. Three of the six patients (cases 3,4 and 6) exhibited enlarged axillary lymph nodes. Local recurrence at the nasopharynx was only observed in case 3 .

Distant metastasis pattern. All the patients exhibited locally and regionally confined disease without synchronous metastasis upon initial diagnosis. All breast metastasis occurred subsequent to treatment as metachronous metastasis. Multiple metastases were the predominant pattern, with distant metastases occurring in other sites in cases 1, 3, 4 and 6 , including in the liver, bone and lung and supraclavicular lymph node. However, the breast was the only site of metastasis in case 2 .

Pathological analysis. The histology of all of the primary nasopharyngeal tumours was poorly differentiated squamous or anaplastic carcinoma. The five documented patients also exhibited anaplastic carcinoma or poorly differentiated squamous cell carcinoma in the breast metastasis, mimicking the primary tumour. IHC and EBV analyses were performed in a number of cases. IHC of the malignant cells revealed negativity for ER and PR in cases 1, 2 and 6, and negativity for HER2 in cases 1 and 2. Positive EBER staining was identified in cases 1,2 and 6 .

Ultrasonographic findings. Ultrasonography was performed for cases 1 and 5. Similar features were observed in the two cases, including a hypoechoic lesion with a heterogeneous internal echo pattern, and no retrotumour acoustic shadowing, microcalcification or skin change. No obvious lymph node enlargement was detected in the two axillary fossa. However, a smooth margin was observed in case 1 while case 5 exhibited an irregular margin.

Treatment strategies and outcomes. Chemotherapy is the first choice treatment strategy if multiple metastases are diagnosed, such as in cases 1,3 and 4. A combination of cisplatin and 5-FU was the standard regimen if the patient was able to tolerate it. However, palliative care was selected if the general situation of the patient was poor, such as for case 6. Radiotherapy was used to reduce the size of the breast metastasis and control the pain associated bone metastasis, as in case 3. By contrast, a Halsted mastectomy was performed with curative intent in case 2 of the current series.

Survival. Median follow-up could not be calculated due to missing data in certain cases. Cases 1 and 3 had short survival 
A

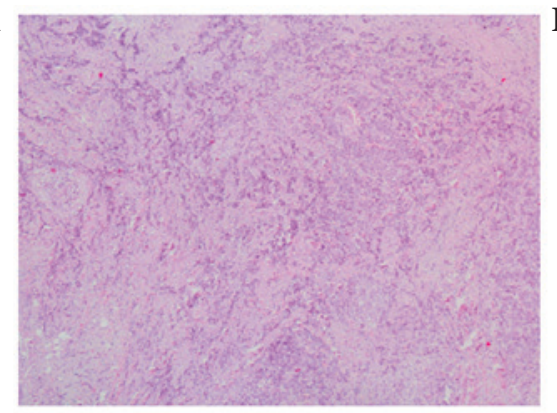

D

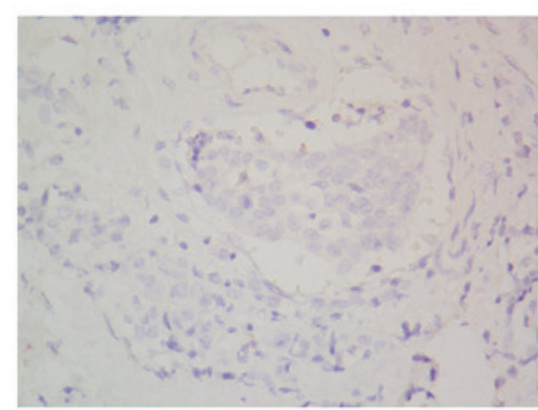

B

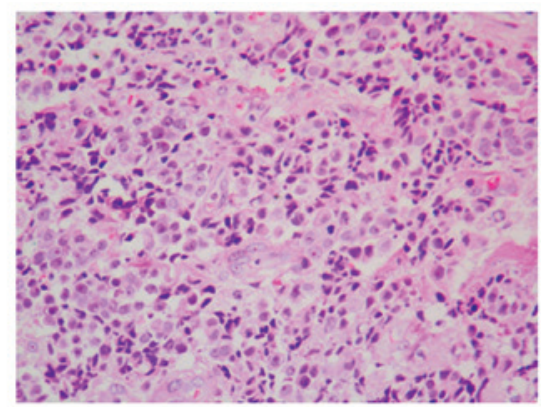

$\mathbf{E}$

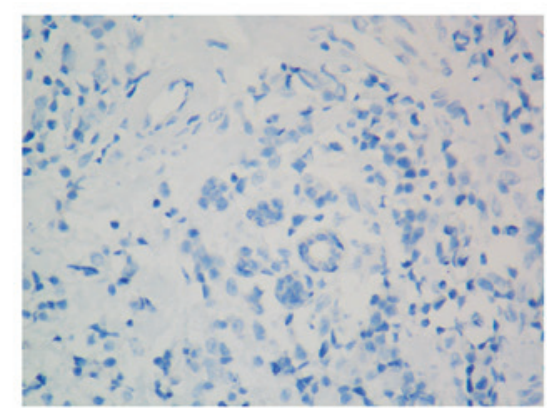

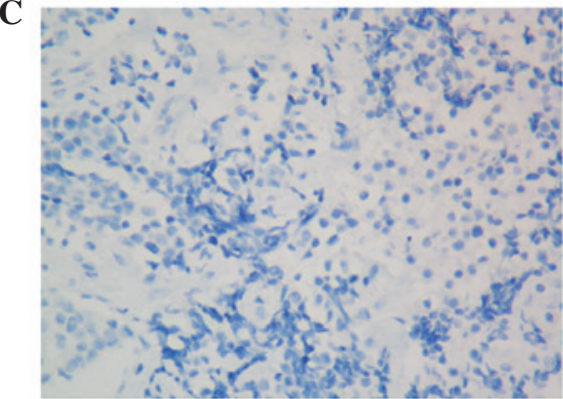

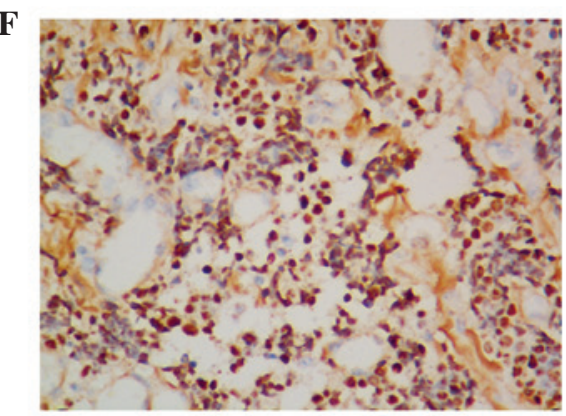

Figure 2. Histological staining of a metastatic breast mass, case 1. Hematoxylin and eosin staining. (A) Anaplastic carcinoma in a nested and trabecular pattern (magnification, x100). (B) Neoplastic cells exhibiting a short spindle or round appearance with little cytoplasm. Immunohistochemical staining using hematoxylin. Tumour cell negativity for (C) estrogen receptor, (D) progesterone receptor and (E) human epidermal growth factor receptor 2. (F) Tumour cell nuclei positivity for Epstein-Barr virus-encoded RNA by in situ hybridization (B-F: Magnification, x400).

A

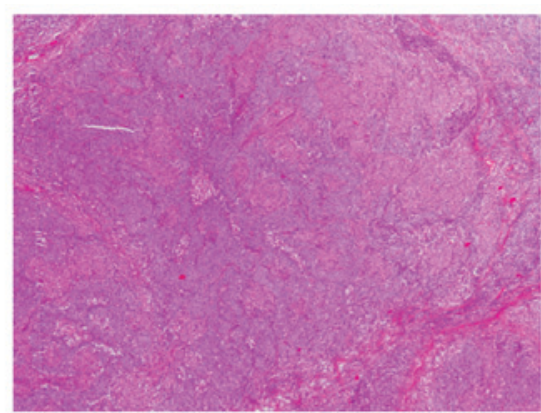

D



B



$\mathbf{E}$

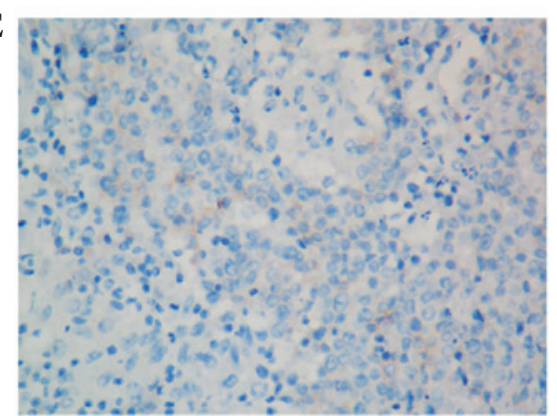

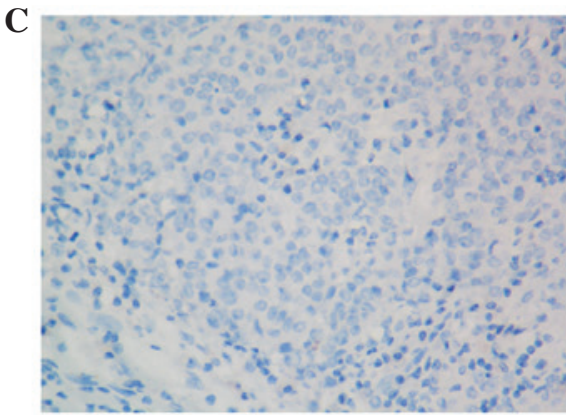



Figure 3. Histological staining of a metastatic breast mass, case 2. Hematoxylin and eosin staining. (A) Poorly and moderately differentiated squamous cell carcinoma diffusely infiltrated in the breast in a nested and trabecular pattern (magnification, x100). (B) Neoplastic cells exhibiting a large round appearance with hyperchromatic nuclei. Immunohistochemical staining using hematoxylin. Tumour cell negativity for (C) estrogen receptor, (D) progesterone receptor and (E) human epidermal growth factor receptor 2. (F) Tumour cell nuclei positivity for Epstein-Barr virus-encoded RNA by in situ hybridization (B-F: Magnification, x400).

times following the diagnosis of metastasis, possibly due to cachexia. The survival times were not reported for cases 4-6; however, their prognosis was considered to be poor due to the presence of multiple metastases. Case 2 from the current series survived without any discomfort for 10 years and was then lost during follow-up.

\section{Discussion}

NPC is a malignant tumour of the nasopharyngeal epithelium with unique epidemiological features, clinical manifestations, treatment strategies and survival outcome (9). It is an endemic disease in Southern China and the incidence of NPC is higher 
in male individuals, with a male:female ratio of 2-3:1 (9). Furthermore, NPC predominantly occurs between the ages of 50-60 years. It is characterised by a tendency to spread regionally to cervical lymph nodes and diffusely to distant organs. Radiotherapy and chemotherapy are the predominant treatment strategies for patients with NPC (2).

Breast metastases from NPC are rare, with 6 cases summarized in the present study (3-5). In contrast to primary NPC, the disease is more frequent among female individuals; however, the median age is similar to that of primary NPC. To the best of our knowledge, case 2 from the current study is the first and only male patient in this clinical setting.

The five female patients (cases 1,3-6) shared the following characteristics: Female, loco-regionally advanced disease at initial diagnosis, metachronous breast metastasis following curative treatment and concurrent disseminated disease at other sites, similar to breast metastasis from other extramammary malignancies (10). The latency period from the initial diagnosis of NPC to the diagnosis of breast metastasis ranged between 6 and 42 months, with a median latency period of 27 months. Breast metastasis may occur in the short term or 2 years after the first course treatment. Case 2 in the current report is unique, as the patient was male. A male patient with breast metastasis from NPC has not previously been reported, highlighting that this disease entity can also occur in males.

The major problem in establishing such a diagnosis is differentiating between primary breast cancer and breast metastasis. Misdiagnosis as a primary breast cancer may lead to an unnecessary mastectomy. Thus, differential diagnosis is vital for ensuring that appropriate chemoradiotherapy is administered for patients with breast metastasis from NPC.

Clinically, breast metastases typically present as a firm mass, may be located at any quadrant and are often superficial. Skin involvement may also exist, while nipple discharge is absent (2). Half of the patients analyzed in the present study demonstrated enlarged axillary lymph nodes. Concomitant local recurrence at the nasopharynx is not frequent; however, distant metastases at other sites are common, including in the lung, liver and bone. These sites are similar to the frequent metastatic sites of primary breast cancer (11). Breast metastases from NPC are rare and typically occur as part of disseminated disease; thus, a previous clinical diagnosis of NPC alone may indicate the metastatic nature of the breast tumour $(12,13)$. The identification of a solitary breast metastasis may also occur, as in case 2. However, ultrasonographic features of breast metastasis are not particularly informative or unique; observations may include regular or irregular margins, hypoechoic lesions, heterogeneous internal echo patterns, an absence of retrotumour acoustic shadowing and no skin changes.

The most important aspect of this differential diagnosis is the pathological morphology and molecular staining of the metastatic lesion. Morphologically, metastatic carcinoma has specific features, characterized by multinodular architecture and a lack of an intraductal carcinoma or lobular neoplasia components. However, Driss et al (5) reported that breast metastasis and primary breast cancer may still exhibit a number of similarities, such as mimicking certain histological types of primary carcinoma, including lymphoepithelioma-like carcinoma, medullary carcinoma or a variety of infiltrating ductal or lobular carcinoma with inflammatory stroma.
Lymphoepithelioma-like carcinoma of the breast may also display multinodular growth. However, Dadmanesh et al (14) identified that lymphoepithelioma-like carcinoma of the breast was not associated with EBV infection, while EBER positivity was identified in the breast metastatic lesions of the three patients tested (cases 1,2 and 6). Thus, ISH is recommended for differential diagnosis. Furthermore, IHC indicated negativity for ER, PR and HER2, a phenotype unique to patients with triple-negative breast cancer. Therefore, pathological, IHC and radiological findings, in conjunction with the patient's clinical history, should be considered in differentiating a secondary mass from a primary breast cancer.

No consensus has yet been reached for the optimum treatment of breast metastasis from NPC. In principle, appropriate chemoradiotherapy or chemotherapy should be administered to patients with multiple metastases, while mastectomy appears unnecessary due to poor prognosis. Radiotherapy may be effective for reducing tumour size as observed in case 3 and may relieve pain in bone metastasis. Supportive treatment should be used for patients with cachexia. The prognoses for these patients is poor, with a survival time of $<6$ months in cases 1 and 3 . The final treatment result was not documented for cases 4-6, however, it is reasonable to predict poor prognosis of these three patients due to the presence of multiple distant metastases.

Breast metastasis was only identified in case 2 and, after receiving radical mastectomy, the patient survived for $\geq 10$ years with no recurrence of disease. In such cases, overall examination and an accurate diagnosis are vital for oncologists to determine the appropriate treatment regimen and predict the patients' survival.

In a patient with a medical history of loco-regionally advanced NPC, metastatic disease should be considered when a breast mass is identified, particularly when the patient is female, with multiple site of malignancies and positivity upon EBER staining. Breast metastasis may also occur in male individuals. Palliative treatment, including chemotherapy and radiotherapy, is the primary treatment strategy for such patients. However, it is considered that patients exhibiting NPC with solitary breast metastasis may still gain long-term survival, therefore, radical mastectomy should be considered.

\section{Acknowledgements}

The present study was funded by the National Natural Science Foundation (grant no. 81372814).

\section{References}

1. Canda AE, Sevinc AI, Kocdor MA, Canda T, Balci P, Saydam S and Harmancioglu O: Metastatic tumours in the breast: A report of 5 cases and review of the literature. Clin Breast Cancer 7: 638-643, 2007.

2. Yeh CN, Lin CH and Chen MF: Characteristics of metastasis in the breast from extramammary malignancies. J Surg Oncol 101: 137-140, 2010.

3. Sham JS and Choy D: Breast metastasis from nasopharyngeal carcinoma. Eur J Surg Oncol 17: 91-93, 1991.

4. Yeh CN, Lin CH and Chen MF: Clinical and ultrasonographic characteristics of breast metastases from extramammary malignancies. Am Surg 70: 287-290, 2004.

5. Driss M, Abid L, Mrad K, Dhouib R, Charfi L, Bouzaein A and Ben Romdhane K: Breast metastases from undifferentiated nasopharyngeal carcinoma. Pathologica 99: 428-430, 2007. 
6. Therasse P, Arbuck SG, Eisenhauer EA, Wanders J, Kaplan RS, Rubinstein L, Verweij J, Van Glabbeke M, van Oosterom AT, Christian MC, et al: New guidelines to evaluate the response to treatment in solid tumours. European organization for research and treatment of cancer, National Cancer Institute of the United States, National Cancer Institute of Canada. J Natl Cancer Inst 92: 205-216, 2000.

7. Li H, Xiao W, Ma J, Zhang Y, Li R, Ye J, Wang X, Zhong X and Wang S: Dual high expression of STAT3 and cyclinD1 is associated with poor prognosis after curative resection of esophageal squamous cell carcinoma. Int J Clin Exp Pathol 7: 7989-7998, 2014.

8. Hong MH, Mai HQ, Min HQ, Ma J, Zhang EP and Cui NJ: A comparison of the Chinese 1992 and fifth-edition International Union Against Cancer staging systems for staging nasopharyngeal carcinoma. Cancer 89: 242-247, 2000.

9. Chua ML, Wee JT, Hui EP, Chan AT. Nasopharyngeal carcinoma. Lancet 2015 [Epub ahead of print].
10. Lee SK, Kim WW, Kim SH, Hur SM, Kim S, Choi JH, Cho EY, Han SY, Hahn BK and Choe JH: Characteristics of metastasis in the breast from extramammary malignancies. J Surg Oncol 101: $137-140,2010$.

11. Marino N, Woditschka S, Reed LT, Nakayama J, Mayer M, Wetzel M and Steeg PS: Breast cancer metastasis: Issues for the personalization of its prevention and treatment. Am J Pathol 183: 1084-1095, 2013.

12. Silverman JF, Feldman PS, Covell JL and Frable WJ: Fine needle aspiration cytology of neoplasms metastatic to the breast. Acta Cytol 31: 291-300, 1987.

13. Domanski HA: Metastases to the breast from extramammary neoplasms. A report of six cases with diagnosis by fine needle aspiration cytology. Acta Cytol 40: 1293-1300, 1996.

14. Dadmanesh F, Peterse JL, Sapino A, Fonelli A and Eusebi V: Lymphoepithelioma-like carcinoma of the breast: Lack of evidence of Epstein-Barr virus infection. Histopathology 38: 54-61, 2001. 